\title{
Touch sensor for social robots and interactive objects affective interaction
}

\author{
Daniele Mazzei ${ }^{1, t} \ddagger$, Carmelo De Maria ${ }^{1, \ddagger}$ and Giovanni Vozzi ${ }^{1,2, *}$ \\ 1 Research Center E. Piaggio, University of Pisa \\ 2 Dipartimento di Ingegneria dell'Informazione, University of Pisa \\ * Correspondence: g.vozzi@centropiaggio.unipi.it; Tel.: +39-050-2217056 \\ + Research Center E. Piaggio, Largo Lucio Lazzarino 1, University of Pisa, 56122 Pisa, Italy \\ $\ddagger \quad$ These authors contributed equally to this work.
}

Academic Editor: name

Version May 18, 2016 submitted to Entropy; Typeset by LTEX using class file mdpi.cls

\begin{abstract}
Recent studies on human-machine and human-robot affective interaction, highlighting the importance of physical experience in empathic exchanges, led to the development of touch sensors for robotics and interactive objects. Most of these sensors are implemented as matrix of pressure sensors. Often of rigid nature they are not suited for all shapes, especially when the device can be subject to deformation. Furthermore, they can usually only capture the pressure without sensing the interaction context which is extremely useful in interaction scenarios. This paper presents a tactile flux sensor able to capture the entire context of the interaction including gestures and patterns. The soft nature of the sensor makes it adaptable to complex and deformable bodies. It is made of successive layers of sensitive and insulating silicone: the sensing layer is obtained by doping the silicone with carbon particles giving it intrinsic piezo-resistive properties. The main features from electrical signals are extracted with the Principal Component Analysis, and a self organising neural network is in charge of the classification and spatial identification of the events, to acknowledge and measure the gesture. The results open to interesting application from toy manufacturing, to human-robot interaction, and even to sport and biomedical equipment and applications.
\end{abstract}

Keywords: tactile interaction; affective robotics; touch sensor; flexible silicone sensor; principal component analysis and neural network; electrical characterisation

PACS: J0101

\section{Introduction}

Affective objects represent an emerging field of human-robot interaction and robotics research focusing on the development of "social intelligence" for machines aimed establishing lifelike empathic relationships with their partners. The term "social intelligence" implies the ability to interact with other people, to interpret and convey emotional signals and to perceive and react to people's intentions with socially and affectively aligned actions [1,2]. Physical shape and embodiment, acting as major affordance [3,4], strongly influence people's expectations inducing the need of a physical contact with affective objects $[5,6]$. Touch and contact experience are amongst the most important affective communication channels used by human beings for empathic exchanges. As human beings we are able to discriminate various touch typologies that strongly correlate with emotions as: anger, fear, disgust, love, gratitude, happiness, sadness and sympathy [7]. Empathic touch is more than a simple analysis of contact point size, position and pressure. Empathic touch requires the recognition of the contact "affective type". Caress or pet are affectively very far from 
pointing touch or slept but very similar if analysed by the point of view of force and pressure applied to the skin. In this paper a soft and stretchable touch sensor for affective objects and social robotics is presented. The sensor is made of silicone allowing an easy integration with other soft material typically used for the fabrication of social robot and "touchable" objects. Together with the sensor, a dedicated signal acquisition electronic board and a signal elaboration algorithm, based on a neural network (NN) were developed, allowing the system to classify the affective category of the perceived touch.

\section{State of the Art}

Affective touch-based interactions between human and robots or interactive objects have recently become an active field of human-robot and human-machine interaction research. The MIT Media Lab team guided by Breazel developed in 2006 Huggable [8]. Huggable was an affective robot endowed with sensitive skin integrated in the robot fur and able to discriminate the type of contact and touch performed by the user interacting with it. In the Haptic Creature Project Yohanan and MacLean [9] designed an animal-like robot with affective haptic sensing able to perceive affective touch. In the last decade the evolution of human-machine interaction has radically changed an underlying assumption that has been in place for centuries: it was up to the humans adapting to know how machines operates, without particular efforts by the machines to support humans in this process. In this new era we are putting humans at the centre of the equation designing a new family of "interactive things" that are invading the affective and empathic fields often considered exclusive to humans [10,11]. Affective touch research has been focused on the design of systems able to endow robots with skins capable of classifying the typology of the perceived interactions [12]. On the other side, touch sensors developed for classic robotics have been designed as systems able to analyse several high resolution parameters of the contact points as force, pressure, size, and vector orientation giving to robots general haptic capabilities [13]. This tendency has been motivated by the recent growth of robotic grasping research aimed at giving to the last generation of robots manipulation capabilities inspired to the human anatomy and cognitive processing. Available tactile sensors usually consist of arrays or touch-sensitive areas, able to generate a contact pressure map. Contact forces and pressures are then used to extrapolate various information related to the physical and topological properties of the contact allowing the analysis of surface properties, the identification of mechanical interactions and of slipping events [14,15]. Many of the latest developed tactile sensors are based on a thin polymer film that acts as a piezo-electric or piezo-resistive detector [16]. Another class of haptic devices is based on magnetic transduction. These sensors exhibit the advantages of having high sensitivity linear behaviour and high robustness [17]. Nevertheless, the design of a system integrating magnetic sensing technology is very complicated and is not always compliant with soft robots and interactive objects. Optical tactile sensors can be also used for touch detection and for torque and applied force measurement. This type of sensors are useful in the detection of slipping detection and for the measurement of its area [18] but are difficult to be integrated in humanoids and social robots. In recent years a new technology for the fabrication of sensorised textiles has also emerged. The production of the sensors on substrates that are not only flexible, but also adaptable to the human body is increasingly widespread. The transduction properties can be obtained by exploiting the intrinsic electromechanical properties of special conductive threads [19]. Alternatively, the detection system is made of rubber doped with carbon black that is used to build sensorial patterns on wearable substrates [20-22]. Sensors developed for robotic grasping and manipulation have usually miniaturized sizes and high spatial resolution in order to be easily embeddable in robotics hands and grippers. These devices can be made of rigid materials covered by soft and elastic layers that partially act as soft interface between the rigid robot structure and the grasped objects. These devices are unfortunately not usable for the fabrication of a new generation of affective objects on which softness and elasticity are considered as main peculiarities. Indeed, socially touchable robots, in order to do not evoke misleading affordances, can't have a rigid or 
semi-rigid touchable interface. For this reason in this paper a novel approach for the fabrication of stretchable silicone-made touch sensitive surface is presented. The designed sensor is based on a matrix topology composed of various sensitivities areas connected to a dedicated electronic driving circuit that acquires and digitizes the signals. A Principal Component Analysis (PCA) is then used to reduce the size of the data and then Kohonen NN for the identification of the touch interaction nature.

\section{Sensor Design and Development}

\subsection{Materials}

Some polymers and plastic compounds possess intrinsic properties of transduction (piezo-resistivity, piezo-electricity, photo-elasticity, magneto-elasticity, etc.), which make these materials particularly suitable for the fabrication of stretchable tactile sensors. The sensor proposed in this work is composed of sensing and insulating layers made of silicone-based materials having similar elastic properties that allow a high mechanical compatibility and integration. The insulating layers are made of Cine-skin Silicone (Burman Company, USA) having a Young modulus which can be modulated between 50 and $250 \mathrm{kPa}$ [21], while the sensing component is made of Elastosil LR 3162 A/B (Wacker Chemie, Italy), a silicone polymer doped with carbon particles having a Young modulus of $5500 \mathrm{kPa}$, according to the producer. The carbon doping confers intrinsic piezo-resistive properties to the silicone without altering the visco-elastic properties.

\subsection{Fabrication Process and Concepts}

The sensor is composed of 3 insulating and 2 sensible layers, for a total of five layers. The sensible layers are characterized by a conductive serpentine structures (Figure 1A), and they are mutually orthogonally oriented. Thanks to this geometry, each sensible layer will provide a coordinate (X or $\mathrm{Y}$ in a Cartesian reference frame) and the projection of the direction (on one of the axis) of the contact event. The deformation of the sensor instead will furnish information about the intensity of the touch. Coupling the signals coming from the layers we will able to infer about vector field magnitude and direction. The bottom substrate is an insulating layer obtained mixing Cine-Skin silicone monomer and catalyser in ratio 10:1 (w/w). In order to obtain a Young Modulus of almost $200 \mathrm{kPa}$ the plasticizer is added in ratio $45 \%(\mathrm{w} / \mathrm{w})$ respect the first solution [21]. Once the three components are mixed, the final solution is degassed under vacuum and then casted in a petri dish in order to have a thickness layer of $2 \mathrm{~mm}$. The layer is then left at room temperature for $24 \mathrm{~h}$ to allow its complete polymerisation. Once the insulating base layer is cured the first conductive serpentine is fabricated. Elastosil monomer is mixed with catalyser in ratio 1:1 ( $\mathrm{w} / \mathrm{w})$ and diluted with $5 \mathrm{ml}$ of trichloroethylene (Sigma-Aldrich, Italy) in order to reduce the solution viscosity allowing a uniform distribution of the polymer during the lithography deposition procedure. The solution is then sonicated for 4 minutes at $0.2 \mathrm{~V}$ obtaining a dense and uniform conductive ink that is finally degassed in a vacuum chamber for 30 minutes. The lithography procedure is performed through the application on the insulating layer of a cellulose acetate mask ( $0.1 \mathrm{~mm}$ in thickness, Figure $1 \mathrm{~B})$ on which the sensor serpentine geometry is impressed through laser cutting technique. Once the mask has tightly adhered to the insulation layer the Elastosil conductive ink is screen-printed using a metallic spatula. The serpentine has a length of $55 \mathrm{~mm}$ with a total width of $14 \mathrm{~mm}$. Each serpentine is composed of 4 lines of $2 \mathrm{~mm}$ in thickness insulated by a $2 \mathrm{~mm}$ space (Figure 1B). After 5 minute, the mask is gently removed and electric wires embedded on the dedicated ending parts of each serpentine. The device is than cured in oven at $50^{\circ} \mathrm{C}$ for $24 \mathrm{~h}$ allowing the complete polymerization of the conductive layer. The second insulating layer, as the first one, is than laid down with a thickness of $1 \mathrm{~mm}$. Once the second insulating layer is cured, another serpentine is built, with orthogonal orientation respect to the first one, using the same technique. Finally the external insulating layer is added with a thickness of $1 \mathrm{~mm}$. The final total thickness of the device is consequently $4 \mathrm{~mm}$. The chosen sandwich geometry, in addition, 
will prevent any possible dispersion of carbon particles from the Elastosil silicon layers: the external surfaces (both top and bottom) are fabricated with the Cine-skin silicone, creating a biocompatible barrier that guarantees users' safety. Figure 1C shows the device in its final configuration with the connection wires.
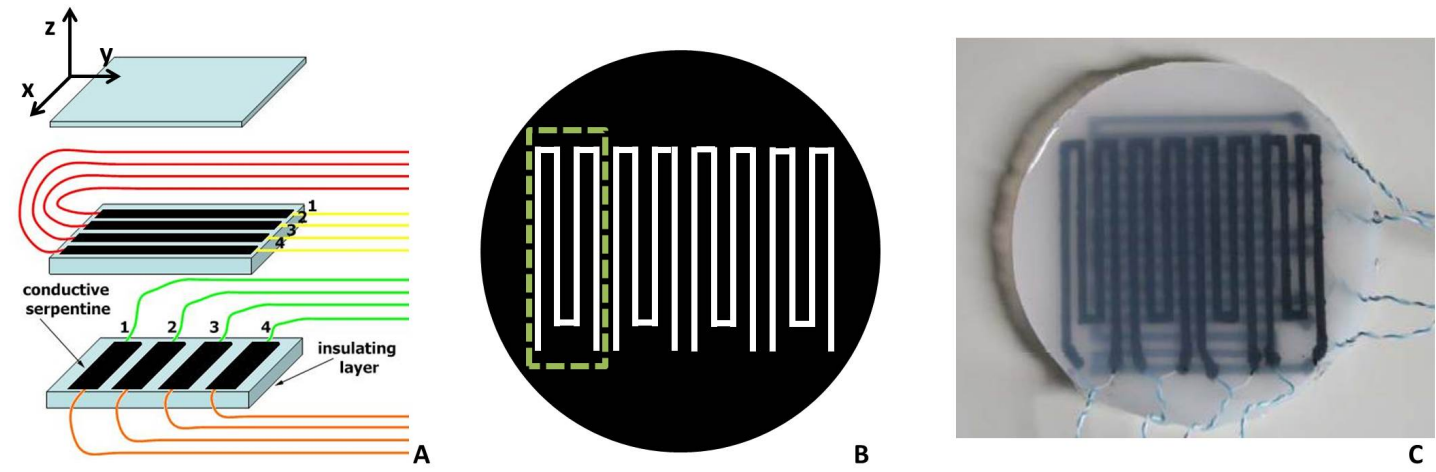

Figure 1. Design principle, structure and topological organization of the sensor (A); cellulose acetate mask used for the serpentine deposition procedure - the geometry of a single serpentine is highlighted by the dashed rectangle (B); final embodiment of the devices, with the connection wiring (C).

\subsection{Electronic driving unit}

The sensible serpentines are made of a piezo-resistive material which acts as a variable resistance. A dedicated electronic driving circuit was designed to drive a constant current in each serpentine integrating 8 trans-conductive voltage driven current generators in a single board. The voltage control input of each current generator is connected to a dedicated buffer amplifier in order to avoid any interference between the various serpentine driving circuits. The inputs of the voltage control buffers are then connected in parallel to an external microcontroller Digital to Analog Converter (DAC) pins allowing the control of the sensor gain. The voltage generated by the current flow on each resistive serpentine is acquired using eight buffer amplifiers, whose outputs are amplified through a non-inverting amplification stage. Each one of these signals is then sent to one of the 8 parallel ADC channels of the micro-controller. An Arduino UNO microcontroller has been used implementing a simple acquisition routine aimed at acquiring at $1 \mathrm{kHz}$ the $810 \mathrm{bit}$ ADC channels. Acquired raw data are streamed through the USB serial connection to the computer on which the data analysis algorithm runs.

\section{Tests and Characterization}

The mechanical behaviour of the sensor was analysed by a cyclic compression test using the Zwick-Roell Z005 uniaxial testing machine with $100 \mathrm{~N}$ load cell. Test protocol consisted of three cycles with a deformation up to $20 \%$ with a strain rate of $10 \% / \mathrm{min}$. The registered stress-strain curves were analysed.

\subsection{Electromechanical characterization}

Electromechanical characterization tests were conducted applying a pressure stimulus and acquiring the sensor signal for 10 seconds in order to analyse the time dependent behaviour induced by deformation impressed on the sensor. Controlled pressure in the range $0-138 \mathrm{kPa}$ was applied in various sensors positions for 0.1 seconds. The resistance of each conductive serpentine was measured before the stimulus application $(t<0)$, at the stimulus application $(t=0.1)$ and then for 10 seconds after the stimulus application $(t=10)$ with a sampling rate of $1 \mathrm{~Hz}$ using a laboratory tester (ICE Strumentazione, Italy). Data was acquired from all the device's channels in parallel in order to analyse stimuli cross influences. Raw resistance data were elaborated extracting various derived parameters 
such as rise time, answer time, recovery time. Due to the goal of applications in affective robotics scenario, in which the temperature of the environment may vary and may also give information about the feeling context, the influence of the environmental temperature on the resistance value was also investigated, performing experiments at several temperatures $\left(10^{\circ} \mathrm{C}, 22^{\circ} \mathrm{C}, 27^{\circ} \mathrm{C}, 30^{\circ} \mathrm{C}, 35^{\circ} \mathrm{C}\right.$ and $\left.50^{\circ} \mathrm{C}\right)$.

\subsection{Signal analysis and touch classification algorithm}

Signals received from the electronic unit are elaborated following the signal analysis protocol illustrated in Figure 2, implemented in a dedicated Matlab ${ }^{\circledR}$ routine (The Mathworks Inc., Massachusetts - US).

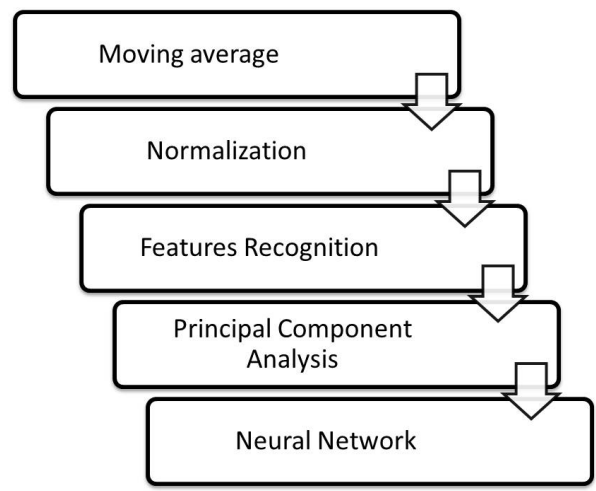

Figure 2. Routine for data analysis.

The first stage is the low pass filtering routine implementing a moving-average filter with sampling window $\mathrm{N}=10$. Each signal is then ratiometrically normalized dividing the actual reading by the signal initial value $\left(\mathrm{V}_{0}\right)$. Thanks to this signal normalisation, the analysis is not influenced by the initial position and stretch of the sensor. $\mathrm{V}_{0}$ is acquired when the system is started and is resampled every minute; such procedure lets the device to accommodate for changes in rest state rejecting input due to robot/object position and environment changes. In order to have spatial information of interaction between user and the device, two new virtual channels, called $5^{\text {th }}$ and $9^{\text {th }}$ Channels, were created, summing the 4 real channels (from top and bottom) at each sampling time. The $5^{\text {th }}$ and $9^{\text {th }}$ channels can be considered as measure of the total sensor layer detected force. For each of the nine channels the following signal features were extracted:

- Maximum Value;

- Maximum Derivative;

- Minimum Derivative;

- Integral over 5 second;

- Steady Value after excitation detection;

- Point in time related to Maximum Value.

For the analysis of touch typology classification, in order to study the sensor capability to be used also in application where very thin skin and surfaces are required, signal coming from the 4 top serpentines only have been used as data input (plus the $5^{\text {th }}$ virtual channel). Moreover, in order to allow the implementation of a very lightweight classification algorithm runnable also in embedded devices the complexity of the inputs was reduced by means of a PCA. PCA matrix was designed in order to keep the information necessary for the identification of the following user-device interaction typologies:

- Touch on zone 1;

- Touch on zone 2; 
- Touch on zone 3;

- Touch on zone 4;

- Caress moving from zone 1 to 4;

- Caress moving from zone 4 to 1 ;

In application where dimensions and computational load are not limited, the described algorithm can be easily extended for the analysis of the bottom layer giving the system the possibility to add an orthogonal analysis dimension. A total of 150 events per type of interaction were recorded creating a PCA input matrix of 900 rows and 30 columns (6 signal extracted features per 5 channels). The extracted principal components (8 components explains the $90 \%$ of variance, see results section) were used as input for the classification stage, implemented as a Kohonen NN. The $\mathrm{NN}$ is characterised by unsupervised training routine and classification layer of $7 \times 7$ neurons; the network weights were initialized through a Gaussian Random Function with values between 0.1 and 0.9. The neighbour radius was initialized as max dimension of network: $r=R_{M A X}=7$. The number of epochs used in the training was set to 1000. During the training phase the winning neuron was selected comparing the outputs of all neurons and choosing the one having the weights vector more similar to the presented input. The neighbourhood effect is introduced by the $\theta(d)$ (eq. 1) which represents the set of neuron whose distance is lower than $r$ from the winning neuron:

$$
\theta(d)=e^{-\frac{\left(x_{k}-x_{w i n}\right)^{2}+\left(y_{k}-y_{w i n}\right)^{2}}{r(t)}}
$$

$r$ is reduced at each epoch, reducing consequently the neighbourhood until it will include the winning neuron only. Once the winning neuron is elected, its synapses and those of neighbour neurons were updated on the basis of their distance from winning neuron using the following law (eq. 2):

$$
w_{k j}(t)=w_{k j}(t-1)+\alpha(t) \theta(d)\left[x_{i j}-w_{k j}(t-1)\right]
$$

where $\alpha$ is the learning rate. Neurons outside the neighbourhood bubble will not have their weights updated. At the end of training phase the space was divided in several regions corresponding to different interaction classes. Each neuron was therefore specialised in recognizing a specific input gesture.

\section{Results and discussion}

\subsection{Mechanical properties}

The result of the compression test on the device is represented in Figure 3, where the stress-strain curves of the three cyclic loads are plotted. It is possible to note the following features:

- the device does not change its mechanical behaviour during cyclic test: i.e. the differences in terms of maximum load (17 $\mathrm{kPa}$ at $20 \%$ of deformation) are less than $3 \%$. This result allows us to infer that the various layers, which compose the sensor, present high mechanical compatibility (no detach or delamination);

- for small deformations (less than $6 \%$, see Figure 3B), and high deformations (more than $12 \%$ ) the device exhibits a linear behaviour, connected by the so-called toe region;

- for small deformation the hysteresis is practically zero, while the loading and unloading phases are different for high deformations, although the difference is constant across the various cycles.

Because it was possible to identify two different regions, we defined two Elastic Moduli as the slope of the linear tracts in these regions: they are equal to $2.8 \pm 0.2 \mathrm{kPa}$ and $232.0 \pm 2.5 \mathrm{kPa}$ respectively. 


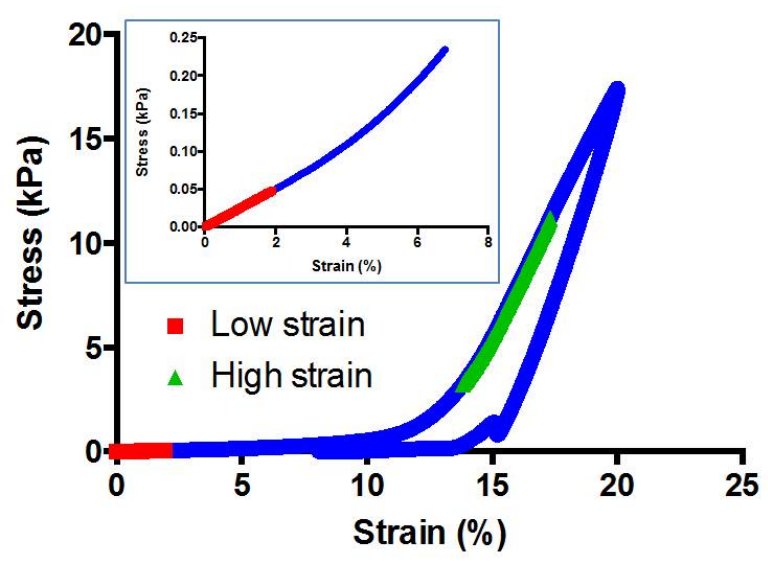

Figure 3. Stress strain curves of the sensor: the three cycles are practically superimposed. In the inset, the stress strain curve for small deformation is presented.

It is interesting to compare these values with those of human skin. The mechanical properties of human skin, a living complex material mainly composed of three layers (epidermis, dermis and hypodermis) [23], whose thickness is a function of age, body zone or hydration, strongly depends on the experimental conditions. In the literature, the Young's modulus of the skin can vary between 0.42 $\mathrm{MPa}$ and 0.85 MPa for torsion tests [24], and between 0.05 MPa and 0.15 MPa for suction tests [25,26]. These values are in the same range of the ones of the proposed sensor.

\subsection{Electromechanical properties}

Data recorded during the pressure stimulation experiment were analysed in order to extract the electromechanical behaviour of the sensor. As shown in Figure 4A-B, the resistance of serpentines increases linearly with applied pressure due to the elongation of the conductive path induced by the stretch of the sensor silicone matrix. The relationship between applied stimulus and serpentine resistance is clearly linear as highlighted in Figure 4B: the linear fitting has an $\mathrm{R}^{2}$ of 0.996 . However the piezo-resistive and visco-elastic properties of the material induce a hysteresis effect which comport a serpentine resistance relaxation time of almost 7 seconds (Figure 4A). Making also in this case a biological comparison, the presented sensor is similar to the slow adapting mechanoreceptors, since it is able to register the interaction and slowly returns to the original position with a behaviour independent from stimulation duration [27]. 


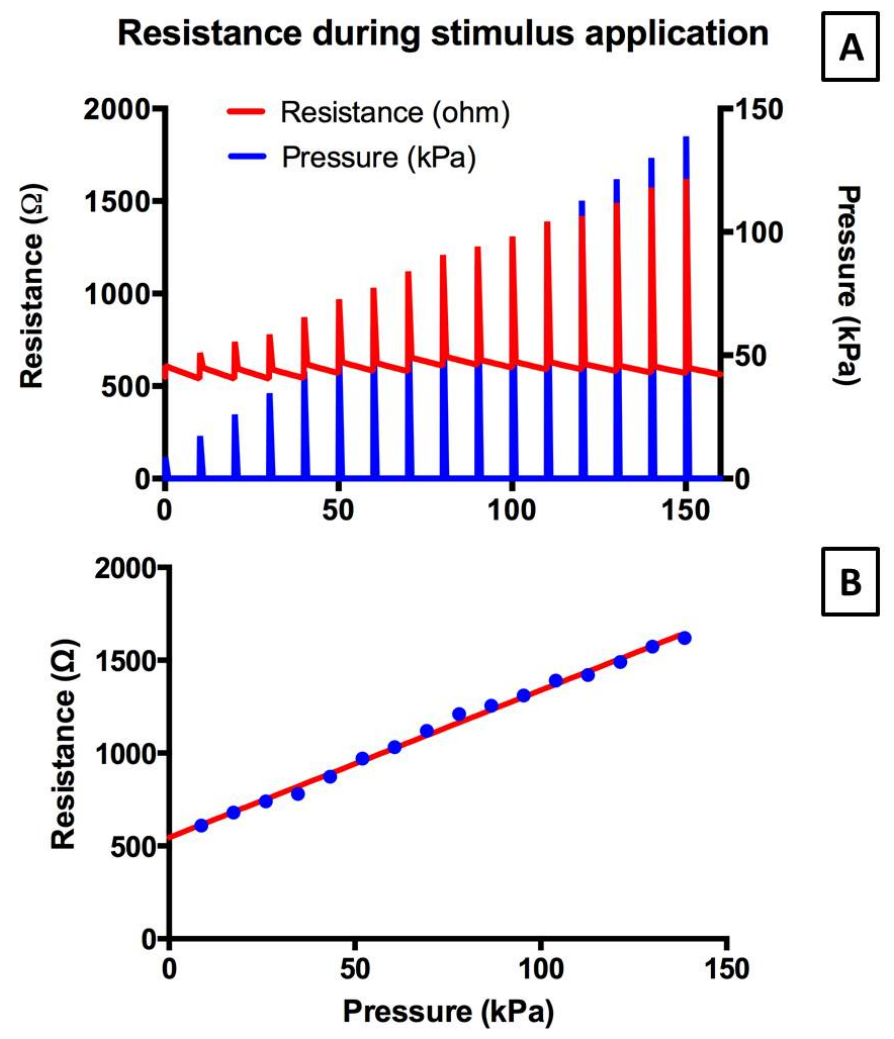

Figure 4. Resistance variation during stimulus application test (A), and the pressure-resistance relationship (B). The test was performed at $22^{\circ} \mathrm{C}$.

Environmental temperature influences the sensor sensitivity linearly increasing the gain from $4.67 \mathrm{Ohm} / \mathrm{kPa}$ to $15.99 \mathrm{Ohm} / \mathrm{kPa}$ with a temperature dependence of $0.28 \mathrm{Ohm} /\left(\mathrm{kPa}{ }^{\circ} \mathrm{C}\right)$ as shown in Figure 5. Moreover, due to the piezo-resistive nature of the device, the baseline resistance also increase with the temperature going from $519.6 \mathrm{Ohm} @ 10^{\circ} \mathrm{C}$ to $560.92 \mathrm{Ohm} @ 50^{\circ} \mathrm{C}$ with an increasing rate of $1.03 \mathrm{Ohm} /{ }^{\circ} \mathrm{C}$.

\section{Resistance peaks at various temperatures}

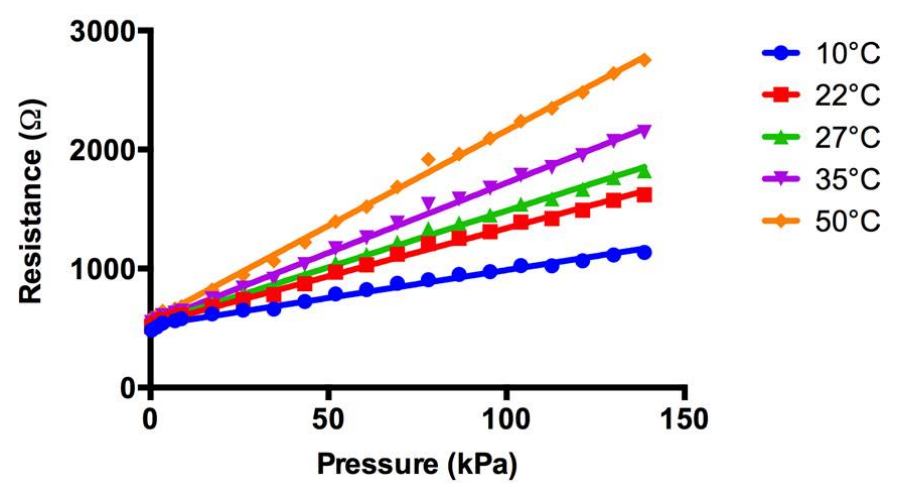

Figure 5. Pressure-resistance relationship at various temperatures.

Stimulus propagation on adjacent serpentines was also measured. For example, as shown in Figure 6A-B, the sensor displays a measurable cross-stimulation effect on both top and bottom perceptive layers, after a stimulus given at zone 1 (Figure 1A). This phenomenon does not prevent the system capability to perceive the touch position, but, on the contrary, it generates, during stimulation, 
a multi signals behaviour that is at the base of the contact typology classification process performed by the implemented NN (see next section).

Top layer

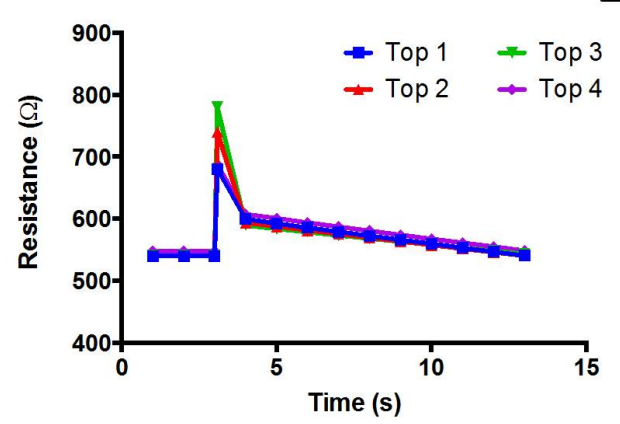

A

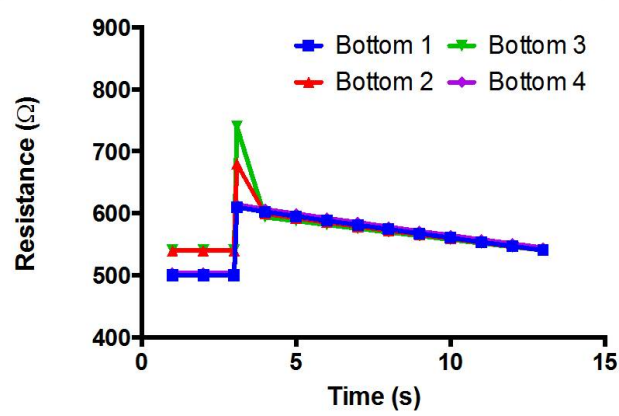

Figure 6. Cross-talk test: the pressure is applied at zone 1, while signals from all channels (of both Top (A) and Bottom (B) layers) are recorded.

\subsection{Affective contact classification}

A typical behaviour of the signals generated by the sensor exhibits a peak with a slow return to the initial value (Figure 6A-B): this behaviour is confirmed also in case of more complex interaction, such as a caress (Figure 7A). From each of the five signals, six different features were extracted (maximum Value, maximum Derivative; minimum Derivative; integral over 5 seconds, steady Value after excitation detection, point in time related to Maximum Value), leading to a total of 30 features.
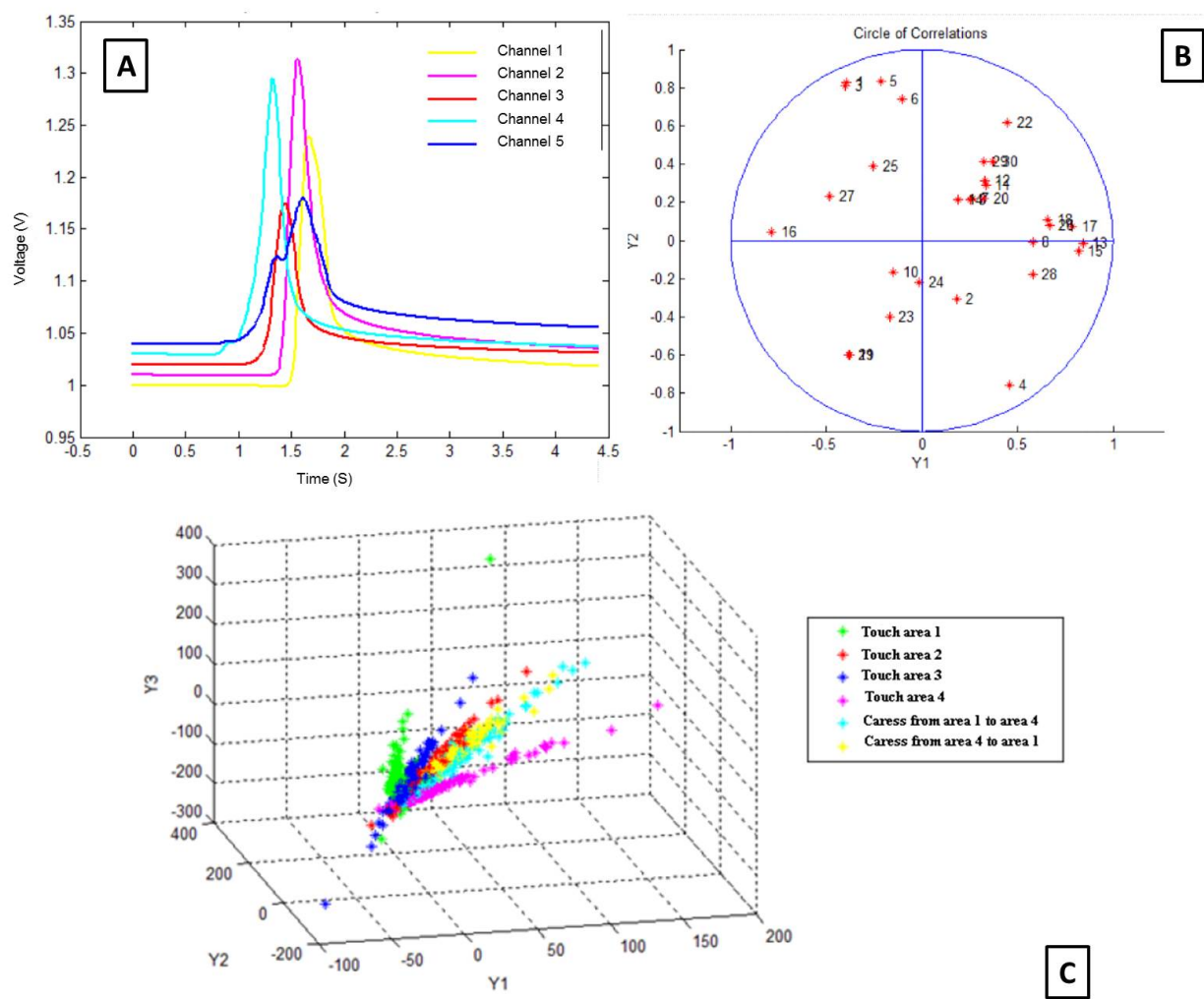

Figure 7. Sensor behaviour during a complex interaction (a caress from zone 1 to zone 4) (A); these types of signals were analysed using the PCA, and described using few parameters: the circle of correlation (B) and the cluster graph (C) indicates the various phases of this analysis. 
In order to simplify the data processing in the NN we used PCA: variance analysis showed that the first 8 Principal Components were able to represent the $90 \%$ of the total dataset variance. Features which are more correlated to the two principal components ( $\mathrm{Y} 1$ and $\mathrm{Y} 2)$ are found by performing a "Circle of Correlations" (Figure 7B). The "Cluster Graph" of Figure 7C was used to see spatial disposition of different classes (types of interaction) into the space where the axes are the first three main components (Y1, Y2 and Y3) (Figure 7C). The NN is in charge to classify 6 different types of interaction (Touch on zone 1, Touch on zone 2, Touch on zone 3, Touch on zone 4, Caress from zone 1 to zone 4 , Caress from zone 4 to zone 1). Following the algorithm presented in materials and methods section, at the end of each training epoch, the error representing the distance between the winning neuron and the input is calculated: in Figure 8A, the maximum and mean classification errors as function of epochs are represented. At the end of the training phase, the space is divided regions, corresponding to the different classes (Figure 8B).
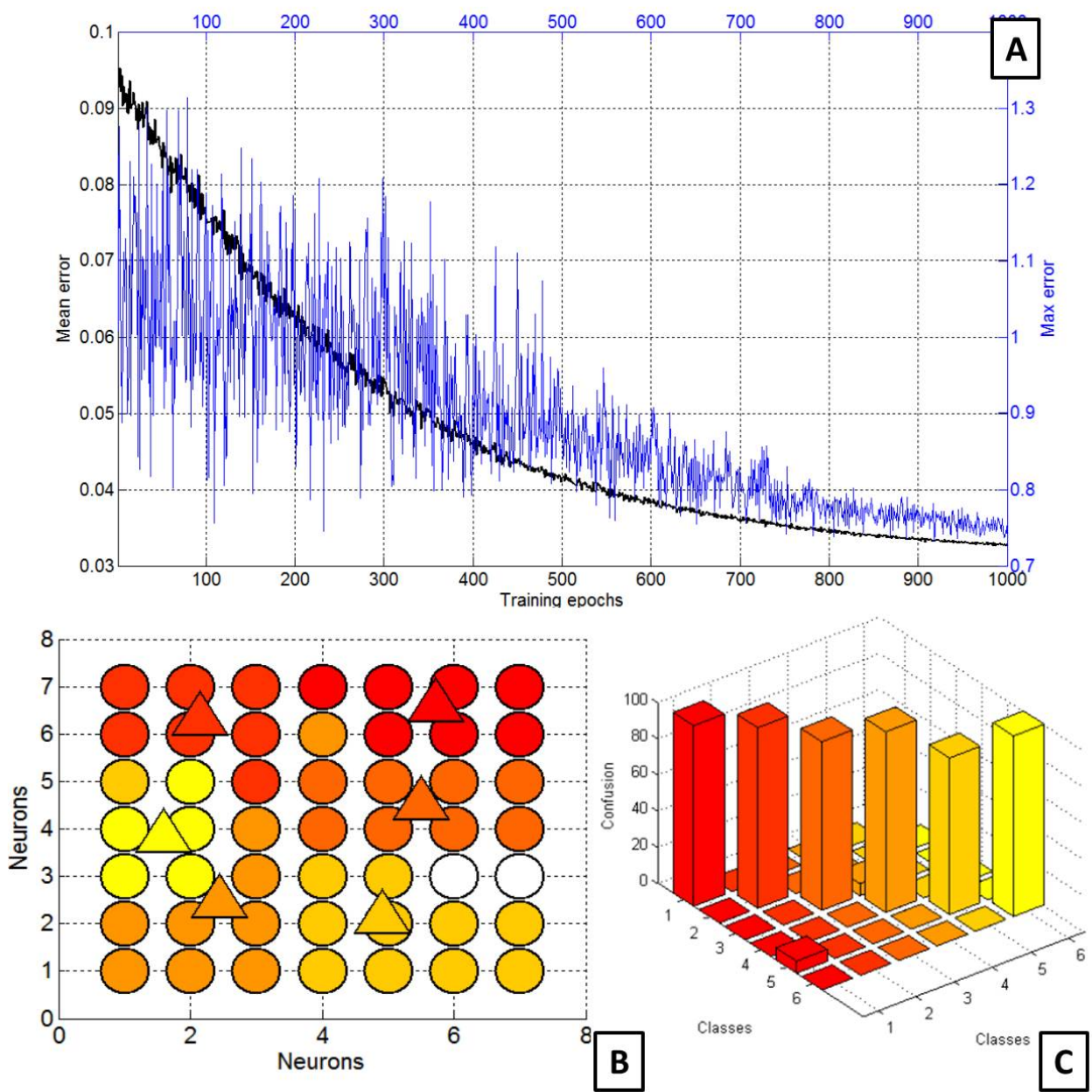

Figure 8. Maximum and average errors during the training phases of the NN (A); subdivision of the $\mathrm{NN}$ into various regions, corresponding to the different classes of interaction (B); confusion matrix (C)

During the "Test Phase" new inputs were presented to the NN without specifying corresponding labels. When classification was completed, it was verified if the network generated the correct result. The confusion matrix (Figure 8C), that gives an index of correct classification, was evaluated. It was a square matrix size equal to number of classes: the rows represent the input classes while the columns the output classes. The classification was correct only if the element along its diagonal were different from zero. With this data processing we were able to discriminate the various kinds of interactions (touch or caress, area of touch and direction of caress), Concluding, the choice of an unsupervised 
learning is due to its ability to extract similarities into complex patterns, working on a big number of real data: a "data intensive" procedure, characterised by more data than computation, is more suitable in applications in which real-time and low computational power are important. A "number crunching" procedure, with several operation on few data, may result in an undesired lag.

\section{Conclusion}

In this paper we presented a novel tactile sensor and the algorithm used to discriminate the different types of integrations, from a simple touch to a caress. The sensor can be seen as a vector field, whose intensity and direction map was analysed by a NN combined with PCA necessary to give meaning to input signals. The dependence of these signals from environmental conditions, such as temperature, was investigated. The comparison with human skin is straightforward: the mechanical properties are very close, and the sensor behaviour can be assimilated to slow adapting human mechanoreceptors. Further research will overcome current sensor limitations, by improving the sensor structure, adding new geometries of the sensing paths, and, of course, faster classification algorithms. Moreover preliminary experiments have been performed integrating fur in the sensors top insulating layer. These experiments shown an increased sensitive of the sensor that can be probably correlated with an amplification effect due to the mechanical torsion of the top layer performed by bended hairs. The developed sensor can be easily manufactured and its production scaled for industrial purposes. The system can be embedded in commercial products allowing the integration of tactile flow sensing in various kinds of objects, for applications ranging from entertainment, to sport, social robotics and thanks to its biocompatibility also to healthcare.

Acknowledgments: This work was partially funded by the European Commission under the 7th Framework Program project EASEL, Expressive Agents for Symbiotic Education and Learning, under Grant 611971-FP7ICT-2013-10.

\section{Bibliography}

1. Breazeal, C. Emotion and Sociable Humanoid Robots. Int. J. Hum.-Comput. Stud. 2003, 59, 119-155.

2. Breazeal, C.L. Designing sociable robots; MIT press, 2004.

3. Gibson, J.J. The concept of affordances. Perceiving, acting, and knowing 1977, pp. 67-82.

4. Horton, T.E.; Chakraborty, A.; St. Amant, R. Affordances for robots: a brief survey. AVANT 2012, 3, 70-84.

5. Feil-Seifer, D.; Mataric, M. Distance-Based Computational Models for Facilitating Robot Interaction with Children. Journal of Human-Robot Interaction 2012, 1, 55-57.

6. Fisher, T.H. What We Touch, Touches Us: Materials, Affects, and Affordances. Design Issues 2004, 20, 20-31.

7. Hertenstein, M.J.; Holmes, R.; McCullough, M.; Dacher, K. The Communication of Emotion via Touch. Emotion 2009, 9, 566-573.

8. Stiehl, W.D.; Breazeal, C.; Han, K.H.; Lieberman, J.; Lalla, L.; Maymin, A.; Salinas, J.; Fuentes, D.; Toscano, R.; Tong, C.H.; Kishore, A.; Berlin, M.; Gray, J. The Huggable: A Therapeutic Robotic Companion for Relational, Affective Touch. ACM SIGGRAPH 2006 Emerging Technologies; ACM: New York, NY, USA, 2006; SIGGRAPH '06.

9. Yohanan, S.; MacLean, K.E. The Haptic Creature Project: Social Human-Robot Interaction through Affective Touch. The Reign of Katz and Dogz, 2nd AISB Symp on the Role of Virtual Creatures in a Computerised Society (AISB '08); Brighton, UK: AISB: Aberdeen, UK, 2008; pp. 7-11.

10. Paiva, A., Affective Interactions: Towards a New Generation of Computer Interfaces; Springer Berlin Heidelberg: Berlin, Heidelberg, 2000; chapter Affective Interactions: Toward a New Generation of Computer Interfaces?, pp. 1-8.

11. Breazeal, C. Toward sociable robots. Robotics and autonomous systems 2003, 42, 167-175.

12. Argall, B.D.; Billard, A.G. A Survey of Tactile Human-Robot Interactions. Robot. Auton. Syst. 2010, 58, 1159-1176.

13. Dahiya, R.S.; Metta, G.; Valle, M.; Sandini, G. Tactile Sensing: From Humans to Humanoids. Trans. Rob. 2010, 26, 1-20. 
14. Loh, K.J.; Azhari, F. Recent advances in skin-inspired sensors enabled by nanotechnology. JOM 2012, 64, 793-801.

15. Tiwana, M.I.; Redmond, S.J.; Lovell, N.H. A review of tactile sensing technologies with applications in biomedical engineering. Sensors and Actuators A: Physical 2012, 179, $17-31$.

16. Lucarotti, C.; Oddo, C.M.; Vitiello, N.; Carrozza, M.C. Synthetic and Bio-Artificial Tactile Sensing: A Review. Sensors 2013, 13, 1435.

17. Nakamoto, H.; Takenawa, S.; Kida, Y. Structure and fundamental evaluation of magnetic type tactile sensor. International Journal of Applied Electromagnetics and Mechanics 2012, 39, 1021-1026.

18. Gu, G.M.; Chang, P.H. Development of a Single-Joint Optical Torque Sensor with One Body Structure. Journal of Institute of Control, Robotics and Systems 2011, 17, 218-222.

19. Yun, D.; Yun, K.S. Woven piezoelectric structure for stretchable energy harvester. Electronics Letters 2013, $49,65-66$.

20. Lorussi, F.; Rocchia, W.; Scilingo, E.; Tognetti, A.; De Rossi, D. Wearable, redundant fabric-based sensor arrays for reconstruction of body segment posture. Sensors Journal, IEEE 2004, 4, 807-818.

21. Wang, S.; Wang, P.; Ding, T. Resistive viscoelasticity of silicone rubber/carbon black composite. Polymer Composites 2011, 32, 29-35.

22. De Rossi, D.; Lorussi, F.; Mazzoldi, A.; Orsini, P.; Scilingo, E.P., Sensors and Sensing in Biology and Engineering; Springer Vienna: Vienna, 2003; chapter Active Dressware: Wearable Kinesthetic Systems, pp. 379-392.

23. Khatyr, F.; Imberdis, C.; Vescovo, P.; Varchon, D.; Lagarde, J.M. Model of the viscoelastic behaviour of skin in vivo and study of anisotropy. Skin Research and Technology 2004, 10, 96-103.

24. Sanders, R. Torsional elasticity of human skin in vivo. Pflugers Arch 1973, 342, 255-260.

25. Diridollou, S.; Patat, F.; Gens, F.; Vaillant, L.; Black, D.; Lagarde, J.M.; Gall, Y.; Berson, M. In vivo model of the mechanical properties of the human skin under suction. Skin Research and Technology 2000, 6, $214-221$.

26. Hendriks, F.M.; Brokken, D.; Van Eemeren, J.T.W.M.; Oomens, C.W.J.; Baaijens, F.P.T.; Horsten, J.B.A.M. A numerical-experimental method to characterize the non-linear mechanical behaviour of human skin. Skin Research and Technology 2003, 9, 274-283.

27. Berne, R.; Levy, M.; Koeppen, B.; Stanton, B. Berne \& Levy Physiology, 6 ed.; Lawrence Erlbaum Associates, Inc.: Philadelphia, PA, 2000. 